\title{
El tahúr y la alcahueta, aliados de juegos prohibidos en Salamanca hacia 1497: Lucena, Repetición de amores $y$ arte de axedre $z^{1}$
}

\author{
María Luisa Gómez-Ivanov \\ Texas State University
}

Repetición de amores se publica en Salamanca hacia 1497 encuadernada junto a un arte de axedrez, obra de un mismo autor conocido por Lucena. ${ }^{2}$ El texto resulta engorroso y confuso para el lector moderno porque el repetidor, un estudiante converso maestro en el juego de ajedrez, parodia tradiciones de amore académicas y cortesanas, mezclándolas y confundiéndolas con el fin de entretener a un público de connoisseurs: los aspirantes a letrado en el recinto universitario. Al presentar la Repetición este carácter singularmente híbrido, primero, por estar a caballo entre lo académico y lo sentimental y, segundo, por su edición con un novísimo juego de ajedrez, cada estudioso en su desconcierto ha subrayado aquella sección o aquel aspecto de la Repetición que juzga más relevante dentro de un discurso amoroso troceado, apenas hilvanado por la voz narrativa del amante

1.- La repetición es un género didáctico ligado a la facultad de cánones y nace de la necesidad de aclarar temas controvertidos para los que el espacio del curso normal no era suficiente. La repetitio, junto con la lectio magister y la disputatio, se proponía la inquisitio veritatis aplicando los tres métodos idénticos pasos exegéticos y exigiendo sobre todo habilidad en el manejo de autoridades. Las repeticiones eran preceptivas una vez al año para los profesores doctores y para los estudiantes suponían el paso previo a la graduación. Se trataba de actos ocasionales pero relativamente frecuentes y marcaban un momento solemne en la vida universitaria. Para el acto de la repetición en la universidad de Salamanca, véase Gómez-Ivanov 2008. Para la edición conjunta de amores y ajedrez en la Castilla de finales del siglo xv, véase Gómez-Ivanov 2005.

2.- Incunable de 124 láminas en octavo ancho sin numerar en el que no consta ni fecha de publicación ni nombre de impresor. Los primeros 73 folios están ocupados por una lección de amores paródico-misógina y los restantes componen un arte de ajedrez dedicado al príncipe don Juan, por lo que su publicación ha de ser anterior a octubre de 1497, fecha en la que fallece el joven heredero. Se trata del primer libro impreso conocido sobre la manera moderna y recién estrenada de jugar al ajedrez todavía vigente y que Lucena bautizó como el ajedrez de la dama. Sobre la importancia de El arte de axedrez de Lucena en la literatura ajedrecista de Occidente véase Pérez de Arriaga 1997: 19. 
rechazado y que al lector de hoy, ajeno a los contextos socioculturales que autorizaron la risa, llega frío, deshumanizado, sin trama ni personajes.

Pese a unas primeras lecturas algo desorientadas y en ocasiones despectivas (Morreale, Ornstein, Matulka, Bussell), la Repetición llegó a despertar más genuino interés por sus afinidades con La Celestina. El entusiasmo de hecho fue tal que algún estudioso aventura que pudiera ser Lucena el secreto autor del primer acto de La Celestina (Cortijo); otro que Rojas se inspiró en Lucena para crear el personaje de Calisto de tal suerte que "Calisto es Lucena» (Calvo 57); incluso se lee que Lucena y Rojas fueron hermanos de sangre (Westerveld). ${ }^{3}$ En todo caso, gracias a esta disposición más acogedora se empezó a comprender en su justo contexto el texto de la Repetición de amores reconociéndosele su mérito como ingenioso remedo burlón de la repetitio salmantinencis, un género docente que se proponía la aclaración de una ley limitándose con frecuencia a la recopilación ad letteram de piezas dispares relacionadas con un tema determinado, sin preocuparse demasiado nunca los repetidores de limar las partes engarzadas. ${ }^{4}$ En cualquier caso, una vez reconocido el género y advertida la parodia la crítica ha identificado los textos engastados en Repetición de amores sin apenas notar cómo el tahúr salmantino interviene en sus originales manipulándolos. El observar cómo el repetidor Lucena, estudiante converso y ajedrecista afamado, se entromete en sus originales retocándolos con segundas y barriendo siempre para dentro permite entrever no sólo inquietudes de su época y de su grupo social, sino también el pulso vital de la Salamanca universitaria de Fernando de Rojas con sus ambientes y personajes clandestinos. ${ }^{5}$

De amore va a dictar el fingido repetidor con la declarada intención de enseñar al hombre a vivir libre del amor a las mujeres y mostrarle sus peligros puesto que «más dañosa te será una muger que ames que mil de que no cures» (Repetición 84). ${ }^{6}$ Sin salirse un ápice de la norma academi-

3.- Govert Westerveld publica estudios rociados de asombrosas genealogías como la que aquí se menciona. En línea pueden encontrarse sus sorprendentes afirmaciones.

4.- Fue este uso del plagio y de adición de fragmentos disformes propios de la repetitio lo que le valió a Lucena y a su obra el desprestigio en la pluma de sus primeros estudiosos pues efectivamente, lejos de su círculo de connoisseurs de las aulas salmantinas y ajeno a los géneros exegéticos de la Edad Media el texto reza como una exposición pedante de fragmentos de amore contradictorios y apenas ligados por la voz narrativa del amante rechazado, "a sort of compendium, an anthology of biblical, classical and medieval lore, traditional argumentation, and contemporary abuse of woman» (Matulka 10), idea desarrollada por Margharita Morreale quien, desconcertada ante el rosario de citas clásicas y la suma amorfa de textos tilda la Repetición de «opera mediocre di mediocre e poco noto autore» (177). Véanse en apéndice los textos engarzados en la repetitio amoris de Lucena con sus fuentes literarias.

5.- Así por ejemplo, Gómez-Ivanov interpreta en su contexto la conclusio altisonante de visos hegemónicos de Lucena, quien a través de un juego discursivo de blancas y negras denuncia burlando desde fuera, en su calidad de converso, a una nobleza, la castellana del siglo xv, anclada a las puertas del Renacimiento en valores medievales arcaicos. Léase Gómez-Ivanov 2007.

6.- Cito por la edición de Fernando Gómez Redondo. 
cista, pues el «orden de mi repetición no difiere del que en las scientíficas letras se usa» (74), Lucena zigzaguea su discurso echando mano, por un lado, de la literatura sentimental que hacía furor en los medios cortesanos de la época y, por otro, del tratadismo amoroso propio del studium. Siguiendo a pies juntillas el esquema formal de la repetitio tras el Preámbulo y el Exordio, Lucena propone el lemma, en principio un punto textual del Digesto que por su dificultad o importancia conviene comentar. Así, a guisa de ley, se infiltra en el coto universitario la primera estrofa del valipendiado poema "Maldezir de las mujeres que fizo Mosén Pedro Torroella» con lo que Lucena introduce en el andamiaje de la repetitio textos de otros círculos que no son los propiamente universitarios, pues el lemma objeto de análisis pertenece al corpus de amore de cualquier amante cortesano rechazado. La mención de Torrellas, cuyas coplas gozaron de gran popularidad y engendraron una ristra de respuestas literarias en cadena despertaría en el público de finales del siglo XV significativas asociaciones extrañas al lector moderno. A partir de este lemma Lucena lanza para su público de escolares un juego de reconocimiento de citas y obras archiconocidas que es a la vez su fórmula de trabajo y su guiño, y que para nada pretende ocultar. ${ }^{7}$

Así tras la improcedente ley a comentar Lucena incluye a modo de exemplum su personal desventura amorosa para que mejor se entienda el libelo antifeminista que será Repetición de amores. Se trata del único segmento propiamente novelesco de la Repetición en el que se narra una recuesta amorosa con ayuda de tercera, parodiando ahora el recurso de la autobiografía en la ficción sentimental pues se trata de "la adaptación de la Historia de doubus amantibus de (Enea Silvio) Piccolomini, que seguramente el público de Lucena recordaba al leerla» (Lacarra 1988: 368). Una vez más Lucena, empeñado en el aplauso y la carcajada, se apropia de un texto tan famoso como polémico. La Historia de duobus amantibus, Euryalo et Lucretia completada en 1444 gozó de una difusión extraordinaria en los siglos XV y XvI. ${ }^{8}$ Según concluye Keith Whinnom resulta imposible tasar el número de ediciones y copias manuscritas que circulaban aunque "some notion of its popularity may be gained from the fact that the British Library catalogues thirty-one editions printed between 1468? and $1566 \ldots$ that the bibliographers who have confined their attention to incunables list thirty-five editions before 1500 , and that it was translated into

7.- Por esta razón no convence la hipótesis de B. Bussell Thompson quien afirma que Repetición de amores tuvo que ser escrita antes de 1495, año en el que se imprime la traducción castellana del De casibus virorum illustrium. "Would Lucena have dared to copy from an accessible and recently printed work of rather major importance? (345). Lucena no esconde que copia pues su artimaña consiste precisamente en la manipulación con segundas de textos bien conocidos.

8.- Sobre el éxito y difusión de Historia de Duobus Amantibus en los siglos XV y xvI véanse Whinnom y Sarriá. 
Italian (twice), French, English, German, and Spanish» (245). El contenido de la novelita italiana más rayano en lo pornográfico que en lo edificante abochornó a su autor quien al entronarse Papa se desvivió por eliminar toda copia a su alcance. Miguel Marciales discute que la traducción castellana de la Historia de doubus amantibus publicada en 1496 en Salamanca pudiera ser obra de Fernando de Rojas, pues efectivamente "se desprende del texto castellano un inconfundible olor y sabor rojano» (Marciales 1618; citado en Whinnon 245). Estos olores y sabores celestinescos del texto latino son los que sedujeron a Lucena quien a la hora de adaptar la sensual novelita de cuna italiana para su público de colegiales salmantinos la sazona con sabrosas pinceladas de color local. Y aquí Lucena no plagia pues efectivamente "la perífrasis del texto de Piccolomini no es tan literal como siempre se ha creído» (F. Gómez 1996: 298). ${ }^{9}$

De la recuesta amorosa que narra Piccolomini Lucena selecciona tres motivos: el retrato físico de la amada, el intercambio de cartas y la intervención de una vieja alcahueta que despierta la respuesta airada de la amada. ${ }^{10}$ Lucena, como acostumbra al apropiarse de otras fuentes, recorta y ciñe, y así condensa doce páginas de la popular novelita latina en sólo tres folios. ${ }^{11}$ No obstante, en la figura de la vieja alcahueta el tahúr salmantino se recrea de lo lindo. La trata familiarmente, la deja hablar, actuar, atemorizarse, caerse, hacer ruido y relacionarse con él y con la amada. Nótense las primeras ampliaciones de la original vieja italiana, fría y descarnada, en manos del estudiante jugador, el converso Lucena. Piccolomini en Italia y Lucena en Salamanca, transidos por las flechas de Cupido deliberan contratar los servicios de una tercera que facilite el trato con la amada. ${ }^{12}$

9.- Así por ejemplo, en 1987 Francisco Márquez Villanueva justifica la omnipresencia de la tercera en las letras peninsulares por motivos socio históricos en su estudio "La Celestina as Hispano-Semitic Anthropology». Pese a advertir el mencionado estudio la presencia de la vieja en la Repetición no se interpreta la recreación castellana del escueto personaje original y así reza: «Lucena looks here, however, as if he were on the footsteps of a similar passage in Piccolomini's Historia de duobus amantibus» (440). Bienvenidos Morros igualmente apunta lo literal del trasvase del texto original al castellano: "Lucena toma como punto de partida una experiencia pseudo-autobiográfica, basada literalmente en la Historia de duobus amantibus de Eneas Silvio Piccolomini, desde la descripción de la amada a la reacción de ésta al recibir la visita de una vieja alcahueta» (299).

10.- Gómez Redondo nos guía para una acertada lectura de Repetición de amores y advirtió antes que yo este detalle (1996: 298-299).

11.- Morros coteja los dos textos de Piccolomini que Lucena incluye en su Repetición y sin dejar de insistir en "la absoluta fidelidad» de Lucena a sus originales (300) demuestra cómo el estudiante "prefiere ser un poco más conciso que su modelo» (302), y si algún cambio introduce es siempre porque o "deja de traducir frases enteras» (305) o decide cortar "por lo sano» (304).

12.- Cotejamos Repetición de amores con la traducción castellana de la Historia impresa en Salamanca en 1496 (ed. Lacertua), «the earlier and probably original edition» (Whinnom 246). 
Historia: determinado enesto, delibró de buscar vna alcayueta con la qual vna carta embíe a Lucrecia. Niso era muy fiel compañero suyo, maestro sagaz de semejantes cosas: éste tomó el cargo y alquiló vna vieja, ala qual encomienda vna carta, la sentencia dela qual fue enla manera siguiente: (45)

Repetición: E como ansí tan súbitamente me hallase d'ella cativo, pensando aliviar la pena comunicándola con alguno, pregunté a una madre mucho mi amiga si la conoscía. E ella, queriendo saber por qué lo dezía, respondíle que, pues su bondad me ponía speranza, y mi buen desseo me dava atrevimiento, que era contento no le negar lo que passava: "Sabrás que me veo assí de llamas encendido, que ni puedo aquesta señora olvidar ni tener algún reposo conmigo, si no me hazes d'ella su conoscido; por tanto, vee, háblale y dile el amor que le tengo». E ella cierto quisiera desviarme el tal pensamiento, si creyera que estuviera en su consejo o en mi libertad; mas, como ya esperimentada, uviesse de otros lo mesmo conoscido, quiso antes aceptar el trabajo con speranza de algún provecho, que ponerse en disputa conmigo; y así, con el esfuerzo que ella me puso, se me encendió el ánimo a tener alguna speranza, por donde, yo con mucho placer, escriví la carta que se sigue: (las cursivas son mías) (77)

Este repetidor aquí no copia al uso propio del género jurídico que parodia sino que explota las posibilidades del texto original animándolo hasta recrear una personalidad nueva, con habla, humanizada, con experiencias y emociones que no aparecen en la «alcayueta» impersonal primera importada de Italia. ${ }^{13} \mathrm{El}$ estudiante salmantino se detiene con la vieja alcahueta y con ella habla a sus anchas porque la conoce bien. Al fin y al cabo, se trata según escribe de "una madre mucho mi amiga» que se interesa por él queriendo saber por qué le hablaba y que le aconseja con objeto de desviarle de arriesgados pensamientos. La vieja es además su primer confidente con quien alivia su "pena comunicándola». Sin embargo, el enamorado Lucena, aunque dice saberla «experimentada de otros» no admite haber disfrutado con anterioridad de sus labores como tercera de amores. Cabría sospechar que tan íntima relación entre el tahúr y la

Aventuro que si alguna traducción tuvo a su alcance Lucena, sería esta edición castellana, más que la latina, para versionarse a gusto del auditorio universitario.

13.- Como advierte Márquez Villanueva «as to the vetula in Aeneas Silvio's Historia de duobus amantibus (a work well known to Fernando de Rojas), she is only a letter-carrier with little else to do and whose services are immediately discarded» (434). 
alcahueta pueda deberse a otro género de servicios, manifiesto en la primera regla del arte de axedrez.

Harold J. Murray afirma en su insuperado estudio The History of Chess que en la Edad media "chess was usually played for a stake... in the Middle Ages the absence of a stake usually calls for remark as something unusual. The stake would ordinarily be of money only» (474-75). No obstante, el juego con apuestas de dinero estaba prohibido en el recinto universitario. Como afirma Murray, "The Universities as a rule took a sterner line than the towns and forbad all knightly occupations, as jousts, hunting and hawking, and chess and games of chance. An exception was sometimes made in the case of chess and tables on festivals and public holidays, on condition that the stakes were limited to eatables and drinkables» (441). Lucena, sin embargo, confiesa en la introducción al arte de axedrez que encuaderna junto con Repetición de amores no sólo haber apostado de manera ocasional, sino conocer de primera mano los peligros de estos envites. Así, en la primera regla de su arte confiesa y advierte: «el que fuere estudiante, créame, porque sé qué es, que si quiere que le aproveche, así para el ingenio como para la memoria, que juegue poco tiempo y el precio sea tan poco que perdido no le pese, porque desta manera alteraría el ingenio y turbaría la memoria» (Ed. Pérez de Arriaga 80). Se entiende que Lucena en más de una ocasión se pasó de la raya apostando y que lo perdido no fue poco pues le pesó; que igualmente no fue poco el tiempo que se dedicaba al juego, afición que practicaba no sólo de día, sino también de noche pues presume de manejar los trucos más nimios para los juegos en penumbras: "si jugáredes de noche con una sola candela, haced si pudiéredes que esté siempre a man izquierda, porque no turba tanto la vista» y por supuesto "aprovecha» una ligera colación pero advierte pues bien lo sabe él "no vino en ninguna manera» (80).

A ojos vistas este estudiante no practicaba el ajedrez en Salamanca como saludable recreación a su principal estudio. Su afición al juego parece más de signo crónico y para ejercitarse y saciar su pasión clandestina, el juego con apuestas de dinero, el tahúr nocherniego tuvo que disponer de un espacio apropiado fuera del terreno universitario. ¿Se desarrollarían estas partidas, hacia 1497, "en los arrabales y allende la puente», cerca de las tenerías, en los dominios que al detalle nos describirá Pármeno de esta «madre mucho mi amiga»? Allí, y según se lee en las Ordenanzas de Salamanca, Lucena encontraría la atmósfera de ganancia ilegítima ya acondicionada por el vino y las mujeres. ${ }^{14}$ ¿Le habría permitido la vieja con "speranza de algún provecho» usar su hacienda para el juego nocturno con apuestas? De tan ducho jugador de ajedrez, aunque amante incipiente,

14.- Sobre la necesidad de reglamentar, conjuntamente, la prostitución, la venta de vinos y el juego en la "casa de la manceuia», a riberas del Tormes, para que allí no se ofendiera «mucho más a nuestro Señor en el pecado», léase Lacarra 1993: 63-73. 
la alcahueta sacaría suculentas tajadas y de ahí su camaradería, su complicidad en la marginalidad del juego, lucrativa delincuencia compartida.

Para llenar de humanidad la figura de aquella "madre» y conferirle protagonismo en la recuesta amorosa el repetidor castellano también aprovecha el episodio de entrega y recepción de la carta.

Historia: Como el alcayueta recibió la carta de Eurialo; luego a más andar se fue para Lucrecia, y hallándola sola, le dixo: «El más noble y principal de toda la corte del César te embía esta carta, y que ayas dél compassion te supplica». (46)

Repetición: La cual carta como rescibiesse aquella madre mía, luego con mucha diligencia se fue a casa de la noble doncella, a la cual hallando sola, dixo: "Aquesta carta te embía un siervo tuyo, suplicándote que d'él ayas piedad». (78)

Historia: Vete luego, hechizera, lleua contigo tu carta; aunque damela, despedacarla he, daré con ella en el fuego». Y arrebatándole la carta, rompióla en pedacos, y acoceada y escopida muchas vezes, dio conella en la ceniza». (46) — después la rescata, "la besó, la puso entre sus joyas y deliberó de escriuir». ${ }^{15}$

Repetición: "iVete de aquí presto, maldita! Y no seas causa de tu muerte que, si alguno aquí te halla, no pensaría quedar satisfecho con ella». Y ansí ella, con el temor que le puso la donzella, toda turbada, sin saber dó ponía los pies, dio consigo de rostros por una escalera do avía subido; de suerte que, tanto por el dolor que de la cayda sentía, cuanto por el daño mayor que esperava, tuvo más cuidado de salvar su vida que de recordarse de la carta. Y como fuese tan grande su caída que a los que estavan arriba pusiesse en saber qué cosa era, abaxó muy presto la donzella y, como no viesse sino sola la carta qu'el súbito miedo de la vieja avía dexado en olvido, acordó tomarla del suelo porque no sospechase el que la hallase más de su inocencia que del atrevimiento de quien scrito la avía. Y como todos seamos desseosos de saber y de atrevimiento nos dé mayor occasión la soledad, buelta a su cámara, comenzó la carta a leer. (78-79) 
La descripción minuciosa de este incidente y la agitación que respira dota al personaje de la vieja de inmediatez llenándolo de vida, de comedia humana. El miedo a la muerte protagoniza esta escena que rezuma celestinesca y es original de Lucena ya que la alcahueta de Piccolomini «sabía de las costumbres de las dueñas» y no hubo temor de la airada reacción de Lucrecia. La recreación literaria del pánico de la alcahueta y de la amada castellana responde a una nueva realidad social que hubo de afectar al enjambre de jóvenes varones que pululaban las aulas salmantinas.

Hacia finales del siglo Xv las autoridades municipales castellanas reconocieron la necesidad de regular el ejercicio de la prostitución por el peligro que suponía para la moral pública la libre circulación en las ciudades de alcahuetas y mujeres sospechosas estableciendo mancebías públicas. ${ }^{16}$ Así, la mancebía de Salamanca, lindante con las bodegas del Tormes "en el arrabal allende el puente» cuyas primeras noticias datan de 1497, se proponía erradicar la prostitución furtiva por lo que las terceras y prostitutas tenían ahora que ejercer su oficio en un espacio confinado vetado a casados, clérigos y estudiantes en el que también se regulaba el despacho de vino y se prohibía el juego. Pese a la nueva reglamentación represiva, la prostitución, el juego y el vino van de la mano y cualquier concentración masculina los hace florecer sea cual fuere la disposición legal que trate de impedirlo. Siendo los arrabales zona de paso frecuente de «viajeros... artesanos, comerciantes, operarios de las industrias del cuero y del grano, ganaderos y rufianes, todos ellos clientes habituales de las mancebías» (Lacarra 1993: 42), cabría sospechar que la vieja no perdiera tan golosa ocasión de escarbar por algún provecho orquestando partidas ilícitas con el más afamado ajedrecista de la zona, el estudiante de leyes Lucena. De ahí la entrañable amistad del tahúr con la alcahueta, aliados en la rentable delincuencia del juego con apuestas en la "casa apartada» de la vieja "cabe el río".

Los alcahuetes desde antiguo habían merecido severos castigos pero a partir de 1497 se incrementaron las penas para quienes practicaran el proxenetismo corrompiendo a mujeres en la ciudad y es en este contexto histórico que Lucena elabora el episodio de entrega de la carta. El miedo a la muerte de la vieja explicitado por Lucena refleja una situación novedosa en Salamanca para las alcahuetas pues ahora les estaba prohibido salir a ejercer libremente por la ciudad y los juicios contra éstas y toda mujer sospechosa se basaban en el conocimiento público del delito, siendo determinante el testimonio de vecinos. De ahí, la elaboración literaria del «temor que le puso la doncella» a la vieja al recordarle la posible "causa de su muerte». De ahí, la precipitación de la vieja por «salvar su vida», preci-

16.- Toda noticia sobre el fenómeno de la prostitución en Salamanca a finales del siglo XV se inspira en los trabajos sobre dicho tema de Eukene Lacarra citados en la bibliografía. Consúltense Lacarra 1993 y 1992. 
pitación que causó tal caída que pudo informar «a los que estavan arriba» de los negocios que se corrían los de abajo con la vieja. De ahí, la presteza de la amada por borrar la huella del paso de la vieja recogiendo la carta del suelo porque sabía que si alguien la encontrara más en entredicho quedaría su inocencia que el atrevimiento del ajedrecista enamorado. El año de publicación del incunable de Lucena coincide con la promulgación de leyes represivas reguladoras de la prostitución en Salamanca y con el establecimiento de mancebías bajo control municipal. Lucena una vez más no pierde oportunidad de traer a colación temas punzantes con los que su público, estudiantes de leyes al corriente de la nueva reglamentación y sin duda clientes de la vieja, simpatizaría. Nótese asimismo cómo la recreación literaria del episodio italiano da cuenta no sólo de la severidad de los recién estrenados castigos con los que se ajusticiaban a las alcahuetas que se andaban con mujeres honradas, sino también del fracaso de los mismos pues efectivamente la vieja seguía ejerciendo sus artes y su labia fuera de su hacienda.

Repetición de amores ilustra igualmente con este episodio de recuesta amorosa la fascinación de la literatura escolar castellana con la alcahueta y su mundo. Tras siglos de convivencia entre judíos, moros y cristianos "Spain first became aware of a situation in which all love, licit or illicit, necessitated the professional services of a third party» (Márquez Villanueva 429) pues así como para los judíos «and, as a matter of principle, matchmaking is for Moslems an honorable, almost religious field of endeavor» (427-428). Si para estas civilizaciones se entiende que todo amor digno de tal nombre, dentro o fuera del matrimonio, se sirve del sabio uso de la experta consumada, Lucena, por su natural converso, habitante de la universidad de Salamanca desde antaño reconocida como capital castellana de la prostitución, no sabría relatar una conquista amorosa como Dios manda sin hacer protagonista de la misma a la mejor tercera, que parece conocía de primerísima mano por otro género de servicio proscripto.

Finalmente el retrato humanizado y familiar de la tercera, único personaje de la Repetición que el ajedrecista trata a sus anchas y con el que se detiene a dialogar respaldaría la hipótesis de la existencia real en Salamanca del personaje Celestina, idea que Peter Russell anima a tomar en serio al advertir cómo los dos autores de la tragicomedia insisten una y otra vez de manera compulsiva en recordarle al lector la existencia de la "casa apartada» que una vez regentó la alcahueta, de óptima arquitectura para toda suerte de actividad criminal.

The Celestina we think of as a wholly original literary creation may in fact be based on the pre-existing character of a real or legendary Salamancan bawd believed once to have lived in the house described by the Portuguese doctor. The constant and apparently pointless 
harping in the text on the exact location of the house in which the bawd used to live becomes entirely explicable if we think of the authors of the book as wishing to establish firmly in the minds of their first readers that the Celestina of their story is based on the character of a real personage whose name... was familiar to all who had studied at Salamanca. (159-160)

Según Russell, el interés de los dos autores por recordarnos la casa en la que Celestina solía vivir- "ieres tú Celestina, la que solía morar a las tenerías, cabe el río? (Celestina 92)-se debe a que existió un personaje real en Salamanca quien regentaba un burdel de fogosas actividades encubiertas y ambos autores esperaban que el público la reconociera situándola en la geografía local. Según Russell «the bawd appears to have the entire establishment to herself. This was, no doubt, to provide secrecy and privacy for her clients, whichever of her various illicit or criminal services it was that they sought» (158). Parece que el ajedrecista nocherniego Lucena disfrutó de la exclusividad de esta propiedad como aliado de la vieja para lucro de ambos, cómplices en la clandestinidad del juego con apuestas en «las tenerías, cabe el río».

Repetición de amores e arte de axedrez se publicó en 1497. Así pues cuando la Comedia de Calixto y Melibea llegó a la imprenta en 1499 ya una vieja Celestina, madre muy amada, andaba en letra impresa, y probablemente vivita y coleando, en Salamanca para regocijo del personal universitario. Si se pudiera especular, animados por Russell, que Rojas y junto a él Lucena recrearon en la ficción a una alcahueta real que ejercía sus variados artes y peligrosos negocios en Salamanca, el retrato del tahúr Lucena se adelantó al de Rojas por un par de años, habiendo adquirido Celestina su primera representación literaria gracias a su valía como corredoras de juegos de mesa ilícitos en la pluma del celebrado ajedrecista Lucena, amigo de Rojas, como él aspirante a letrado y converso. ${ }^{17}$

\section{Apéndice}

\begin{tabular}{|l|l|}
\hline \multicolumn{1}{|c|}{ Partes } & \multicolumn{1}{c|}{ Fuentes literarias } \\
\hline PREAMBULO: Dedicatoria a su amiga & Tradiciones cancioneril y sentimental \\
\hline EXORDIO: «Preclaríssimas señoras» & Tradición burlesca estudiantil \\
\hline -Elogio de la castidad & Tradición del elogio a mujeres \\
\hline -Elogio de la virtud & Escritos éticos \\
\hline
\end{tabular}

17.- Sobre posibles relaciones entre Rojas y la familia Lucena léanse Calvo, Keats, Westenberg y Cortijo. 


\begin{tabular}{|l|l|}
\hline LEMMA: El Maldezir de Torrellas & El Cancionero \\
\hline -Desaventura amorosa de Lucena & Historia de duobus amantibus \\
\hline -Omnipotencia del Amor-Eros & Tratado de como al home es necesario amar \\
\hline -Descripción de Cúpido & Diez questiones del Tostado \\
\hline EL NOTABLE : demostración de la tesis & Mulier est hominis confusio \\
\hline -El libro albedrío y la virtud & Ethica a Nicómaco \\
\hline -Reprobación del amor mundano & De amoris remedio (Piccolomini) \\
\hline -La natura femenina falsa y lúbrica & Boccaccio, Juvenal, Pseudo-Aristóteles \\
\hline -Retracción pública & Palinodia misógina \\
\hline LA CONCLUSION: Cuestión amorosa & Debate entre el clérigo y el caballero \\
\hline -Primacía de las letras sobre las armas & Topos Sapientia vs Fortitudo \\
\hline -Primacía de las armas sobre las letras & \\
\hline FIN: Elogio a su amiga & \\
\hline $\begin{array}{l}\text {-Petición de favores por el servicio } \\
\text { prestado }\end{array}$ & Tradición cancioneril y sentimental \\
\hline
\end{tabular}

\section{Bibliografía}

Calvo, Ricardo. Lucena: La evasión en ajedrez del converso Calisto. Ciudad Real: Perea, 1997.

Cortijo Ocaña, Antonio. "An Inane-Hypothesis: Torroella, Flores, Lucena, and Celestina?» En Proceedings of the Comparative Literature, Linguistics and Culture: An Iberian Dialogue. Berkeley: The Gaspar de Portolà Catalonian Studies Program, 1977: 40-56.

Gómez-Ivanov, María Luisa. "La encuadernación del incunable de Lucena, Repetición de amores e arte de axedrez: con CL juegos de partido." Medievalia 37 (2005): 39-51.

- "Pedagogía festiva en Salamanca, hacia 1497: Luis de Lucena y la Repetición de amores.» RILCE: Revista de Filología Hispánica 24 (2008): 65-79.

-. "El Humanismo castellano en el ingenio perspicaz del converso Lucena: Cedant arma toge (Salamanca, h.1497)». Romance Notes 47:2 (2007): 125-32.

Gómez Redondo, Fernando. "Lucena, Repetición de amores: sentido y estructura». En "Nunca fue pena mayor." Estudios de literatura española en homenaje a Brian Dutton. Cuenca: Universidad de Castilla-La Mancha, 1996: 293-304.

—. Ed. Lucena, "Repetición de amores». Madrid: Universidad de Alcalá, 2014. Keats, Victor. Chess, Jews and History. Oxford: Oxford Academy Publishers, 1994. 
LACARRA, Eukene. «Sobre la cuestión de la autobiografía en la ficción sentimental». En Actas del I Congreso de la Asociación Hispánica de Literatura Medieval. Ed. Vicente Beltrán. Barcelona: PPU, 1988: 359-68.

- " «a evolución de la prostitución en la Castilla del siglo xV y la mancebía de Salamanca en tiempos de Fernando de Rojas». Fernando de Rojas and Celestina: Approaching the Fifth Centenary. Madison: Seminary of Medieval Studies, 1993: 33-78.

-. "El fenómeno de la prostitución y sus conexiones con La Celestina.» Historias y Ficciones. Coloquio sobre la Literatura del Siglo XV. Valencia: Universitat de València, 1992: 267-278.

-. Ed. La Celestina. Madison, 1995.

Márouez Villanueva, Francisco. "La Celestina as Hispano-Semitic Anthropology». Revue de littérature comparée 4 (1987): 425-53.

MatUlKa, Barbara. "An Anti-Feminist Treatise of Fifteenth Century Spain; Lucena's Repetición de amores». Romanic Review 22 (1931): 99-116.

Morreale, Margharita. "La Repetición de amores di Luis de Lucena: alcuni aspetti della prosa spagnola del Quattrocento». Quaderni Ibero-Americani 3 (1956): 177-181.

Morros, Bienvenido. "Piccolomini y la Repetición de amores». Revista de filología hispánica LXxxiII (2003): 299-309.

MurRaY, Harold J.R. A History of Chess. Oxford: Clarendon Press, 1913.

Ornstein, Jacob, ed. Repetición de amores. Por Luis de Lucena. Chapel Hill: University of North Caroline Press, 1954.

- "La misoginia y el profeminismo en la literatura castellana». Revista de Filología Hispánica 3 (1941): 219-32.

Pérez de Arriaga, Joaquín. El incunable de Lucena: Primer arte de ajedrez moderno. Madrid: Polifemo, 1997.

Piccolomini, Aeneas Sylvius. Estoria muy verdadera de dos amantes Eurialo Franco y Lucrecia Senesa, Travaux et Mémoires. Ed. Jean Paul Lecertua. Etudes Ibériques, Vol. I. Limoges: U.E.R des Lettres et des Sciences Humaines de Limoges, 1975: 1-78.

Russell, Peter. «Why did Celestina Move House?» The Age of the Catholic Monarchs, 1474-1516. Literary Studies in Memory of Keith Whinnom. Ed. Alan Deyermond and Ian Macpherson. Liverpool University Press: 155-161.

Sarrí́ Rueda, Amalia. "Ediciones del siglo XVI en castellano de Historia de duobus amantibus». El libro antiguo español: Actas del primer coloquio internacional. Salamanca: Universidad de Salamanca, 1988: 345-59.

Thompson, B. Bussell. "Another source for Lucena's Repetición de amores.» Hispanic Review 45 (1977): 337-345.

Whinnom, Keith. "The Historia de duobus amantibus of Aeneas Sylvius Piccolomini (Pope Pius II) and the Development of Spanish Golden-Age Fiction." Essays on Narrative Fiction in the Iberian Peninsula in Honour of Frank Pierce. Ed. Robert B. Tate. Oxford: Dolphin, 1982: 243-55. 

Gómez-Ivanov, María Luisa, «El tahúr y la alcahueta, aliados de juegos prohibidos en Salamanca hacia 1497: Lucena, Repetición de amores y arte de axedrez", Celestinesca 39 (2015), pp. 39-52.

\section{RESUMEN}

Lucena, autor de Repetición de amores y arte de axedrez, fue un ajedrecista afamado de origen converso. Pese a ser su Repetición una obra fría, descarnada, propia del género que manipula, el célebre ajedrecista, estudiante de leyes en Salamanca, se detiene a recrear su amistad con una vieja, buena amiga, que parece conocía de primera mano. Este artículo interpreta la relación entre el tahúr y la alcahueta con intención de respaldar la hipótesis de la existencia real en Salamanca de una vieja Celestina, cuyas primeras artimañas impresas en 1497 se debieron no tanto a sus artes en la tercería ilícita de amores sino sobre todo a su labor como corredora de lucrativos juegos de mesa clandestinos.

PALABRAS ClAVE: Celestina avant la lettre, ajedrez, juegos, converso, pedagogía.

\section{ABSTRACT}

Lucena, the author of Repeticion de amores y arte de axedrez, was a well-known chess player and a converso. While his Repetición reads as a cold, lifeless text characteristic of the academic genre he is mocking, within its lines the celebrated chess player and law student at Salamanca spends some time recreating his close friendship with an old woman famed among students as an adept go-between. This article delves into de close relationship between the gambler and the bawd with the intention of supporting the hypothesis of the existence in Salamanca of a real woman whose secret services, first recorded in 1497, included not only illegal matchmaking but also the running of lucrative illicit table games.

KEY WORDS: Celestina avant la lettre, chess, gambling, converso, pedagogy.

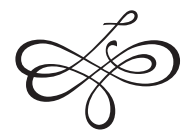

